\title{
Mark-Georg Dehrmann
}

\section{Dürrenmatt in Delphi Korrekturen des Ödipus-Mythos im Sterben der Pythia ${ }^{1}$}

Der junge Ödipus, so berichtet uns Dürrenmatt in seinem 1976 erschienenen Sterben der Pythia, ${ }^{2}$ ist nicht ahnungslos zum Orakel gegangen, nein, er wusste, dass er als Findelkind nach Korinth gekommen war. Der Jüngling wollte Apollon mit einer falschen Frage herausfordern. Dessen Spruch gläubig annehmend, erfüllte er in heiliger Raserei das Verheißene, tötete Laios und heiratete Iokaste. Durch das Pestorakel, das die Aufdeckung des alten Mordes forderte, verstand er endlich den Sinn des verhängten Schicksals: Die Götter hatten ihn, indem er ihren Willen erfüllte, vernichten wollen. Im Triumph führte er den Prozess gegen sich selbst, und im Triumph verstümmelte er sich. Triumphierend schließlich zog er als Bettler umher.

Man kann Dürrenmatts an anderer Stelle ausgesprochene Deutung des Sophokleischen Ödipus - dass ,ein unbewusst Schuldiger von einem unbarmherzigen Gott gezwungen [wird], sein eigener Richter zu sein" ${ }^{\circ 3}$ - teilen oder nicht, seine eigene Bearbeitung der Gestalt befremdet zunächst. Das mag nicht nur an der extremen Psychologie der Figur liegen, sondern auch daran, dass das Vorangehende erst einen kleinen Ausschnitt der viel verwickelteren Geschichte darstellt. Um eine Perspektive auf Dürrenmatts Mythenkorrektur zu gewinnen, bietet es sich jedoch an, vorerst die weitere Handlung auszublenden und die Figur des Ödipus ins Zentrum zu stellen.

Dass es sich bei der skizzierten Version um eine Mythenkorrektur handelt, ist auf den ersten Blick evident. Während der mythische Ödipus nach Aristote-

1 Für Kritik, Anregungen und produktive Gespräche danke ich Antje Wessels und Martin Vöhler.

Der Aufsatz wurde für die Online-Publikation geringfügig überarbeitet. Zuerst erschienen ist er in: Mythenkorrekturen. Zu einer paradoxalen Form der Mythenrezeption. Hrsg. von Martin Vöhler und Bernd Seidensticker in Zusammenarbeit mit Wolfgang Emmerich. Berlin u. New York 2005, 401-409. Die Originalpaginierung wurde beibehalten.

2 Das Sterben der Pythia ist Teil des Nachworts zum Nachwort der Komödie Der Mitmacher (Zürich 1976). Nachweise erscheinen fortlaufend im Text und beziehen sich auf die Neufassung für die Werkausgabe, erstmals erschienen Zürich 1980. Literatur zum Sterben der Pythia: Heinz Schmitz: Oedipus bei Dürrenmatt. Zur Erzählung „Das Sterben der Pythia“. In: Gymnasium 92 (1985), S. 199-208. Leider nicht greifbar war mir der Aufsatz von Ulrich Weber: Déconstruction du mythe d'Edipe et tragédie du Minotaure. Friedrich Dürrenmatt et la mythologie grecque. In: Mythe et Science. Actes du colloque du 14 au 16 mars 2002, Neuchâtel, Suisse, hrsg. von Andreas Dettwiler und Clairette Karakash. Lausanne 2003, S. 115 25.

3 Friedrich Dürrenmatt: Nachwort zu Achterloo IV. In: Ders.: Gedankenfuge. Zürich 1992, S 144 f. Ähnlich auch F. Dürrenmatt: Prometheus. In: Ders. (1992), S. 7. 
les handelt, „ohne die Furchtbarkeit der Handlung zu erkennen“, ${ }^{4}$ begeht Dürrenmatts Ödipus seine Verbrechen wissend. Aus der Tragödie des Unwissens wird ein Drama des Wissens, und zwar in einem umfassenden Sinne, der sich nicht nur auf die Figur des Ödipus, sondern ebenso auf das zahlreiche andere Personal von Dürrenmatts Text bezieht.

Da für das Wirken des Schicksals, wie es Sophokles' Drama zugrunde liegt, Ödipus' Unwissen aber konstitutiv ist, dreht sich, wenn Ödipus weiß und trotzdem handelt, die Priorität zwischen Schicksal und Wille um: Ödipus, so wird man sagen müssen, will das als Schicksal Verhängte erfüllen. Dem entspricht, dass Dürrenmatt angibt, Ödipus dem Schicksal „entreißen“ (273) und die Geschichte vom Zufall her erzählen zu wollen. Wissen statt Unwissen, Zufall statt Schicksal - so lauten die Gesichtspunkte, die den ersten Teil der folgenden Untersuchung leiten sollen.

Der zweite Teil zielt auf die Bedeutung der Mythenkorrektur im Sterben der Pythia. Sieht man genauer auf die referierte Ödipus-Variante, so muss man sich Sophokles' und Dürrenmatts Fassung als mythos im Aristotelischen Sinne identisch vorstellen: Auch wenn Ödipus seine Furcht spielt, sein Erstaunen inszeniert, wenn seine Analyse ein - zugegeben fürchterlicher - Coup de théatre ist, bleibt doch die äußere Handlung gleich. ${ }^{5}$ Wir stehen vor dem paradoxen Befund, dass der Mythos, obwohl er in seinem Kern verändert wird, auf seiner Handlungsgestalt beharrt, dass sich trotz Wissens und Zufalls der tragische Ausgang der Geschichte behauptet. Die alte Geschichte scheint bei Dürrenmatt einen modernen Sinn ergeben zu können, für den es genügt, die Prämissen, nicht jedoch die Fatalität des Verlaufs zu korrigieren.

4 Aristot. Poet. 14. Zitiert nach Aristoteles: Poetik, übers. von Manfred Fuhrmann. Stuttgart 1982, S. 45.

5 Dächte man sich Dürrenmatts Fassung der Ödipus-Handlung als Drama, so müsste man sie sich von außen betrachtet als absolut identisch mit dem Geschehen denken, das Sophokles auf die Bühne stellt. Ja, man könnte den Oidipus Tyrannos selbst durchaus mit der Dürrenmattschen Annahme eines geheimen Wissens seines Helden lesen, ohne dass inhaltliche Widersprüche entstünden. Dürrenmatt beschreibt diese Verdopplung eines Stoffs durch die Veränderung des Bewusstseins, nicht jedoch der Handlungen seines Protagonisten als ironische Literatur: „Angenommen, Don Quijote wäre ein ironischer Held, so würde dieser Don Quijote zwar ebenfalls gegen eine Windmühle und gegen eine Schafherde anrennen, aber er würde wissen, daß diese Windmühle eine Windmühle ist und kein Riese und die Schafherde eine Schafherde und kein Ritterheer; seine Ironie bestünde darin, daß er so täte, als hielte er die Windmühle für einen Riesen und die Schafherde für ein Ritterheer. [...] Demnach gibt es zwei Literaturen, eine ,wirkliche' und eine ,ironische', das heißt, daß jedes Buch gleichsam zweimal existiert, in zwei wörtlich identischen Texten gleichen Inhalts [...]“ (204-206). Vgl. zur Ironie des späten Dürrenmatt und ihren Quellen bei Kierkegaard: Ulrich Weber: „Ob man sich selbst ein Stoff zu werden vermag?“ Kierkegaard und die Entstehung des subjektiven Schreibens im Mitmacher-Komplex. In: Quarto 7 (1996), S. 65-79. 


\section{Drama des Wissens und des Zufalls}

Zunächst muss klargestellt werden: Das Sterben der Pythia ist kein Theaterstück, sondern eine Erzählung. Wie schon der Titel sagt, wird die Geschichte nicht aus Ödipus' Perspektive erzählt, sondern aus der der Delphischen Pythia Pannychis. Diese ist, was Henry James „ficelle“ nennt, eine Figur, die eingefügt wird, um den anderen Charakteren ihre Rede zu entlocken. Pannychis, verantwortlich für das berühmte Orakel, erfährt nach dem Ödipus-Geschehen, das wir von Sophokles kennen und das sich bei Dürrenmatt hinter der Erzählbühne abspielt, dass sich ihr Spruch erfüllt hat. Als sie sich zum Sterben niederlegt, treten ihr die Beteiligten als Schatten entgegen, um der Priesterin jeweils ihren Anteil an der Geschichte zu berichten: zuerst Menoikeus, dann Laios, Ödipus, Iokaste, Tiresias, gefolgt von der Sphinx und noch einmal Tiresias. Mit jedem Bericht enthüllen sich neue, unvermutete Hintergründe der Geschichte, bis die Pythia schließlich selbst in das Totenreich eingeht. Hinter das analytische Drama des Sophokles, das das Wirken des Schicksals enthüllt, wird als Analyse des schicksalhaft Erscheinenden ein LukianischWielandsches Totengespräch geblendet. Die Tragödie wird durch den epischen Bericht ihrer Hintergründe dekonstruiert. Die Frage, die die Pythia sich stellt, ist auch die des Lesers: Wie konnte das passieren?

Denn: - Pannychis' Orakel kommen nicht von Apollon. Sie sind freie Erfindungen eines äußerst erfolgreichen Wahrsagebetriebs. Und obwohl manchmal ein Spruch von zahlungskräftigen Hintermännern in Auftrag gegeben wird, war dieses bei dem Mord- und Inzestorakel doch nicht der Fall. Dem adligen Jüngling, der plötzlich vor ihr erschien, wollte die Priesterin vielmehr durch ein möglichst abwegiges Orakel den Götterglauben austreiben. Einer „,schlechtgelaunten Pythia“ fällt also, wie Dürrenmatt die Erzählung einleitet, Ödipus „Zum Opfer“ (274). Nachdem dann Pannychis erfahren hat, dass sich unwahrscheinlicherweise ihr Orakel erfüllt hat, beginnt sie, die Vorgeschichte des Vorfalls zu rekonstruieren. Im Archiv stößt sie auf ein erstes Orakel, das ihre Vorgängerin dem Laios verkündet hat: „Werde ihm ein Sohn geboren, werde dieser ihn ermorden“ (282). Tiresias, der immer mal wieder für mächtige Kunden Sprüche bestellt, gab den Auftrag dazu, in diesem Fall für den Drachenmann Menoikeus. Offensichtlich sollte das Orakel dessen Sohn Kreon auf den Thron bringen, indem es verhinderte, dass der verhasste Laios einen Nachkommen zeugt. Gleichzeitig fällt der Pythia ein anderes, kürzlich von ihr widerwillig ausgerichtetes Orakel ein, das, ebenfalls von Tiresias bestellt, für die Entlarvung von Laios' Mörder das Ende der Pest in Theben in Aussicht gestellt hatte. Während also das erste und das dritte Orakel offensichtlich korrupten Machenschaften dienten, war doch ihres fast gänzlich ohne Hintergedanken in einer üblen Laune ausgesprochen. Wie also konnte es passieren, dass das zufällige Orakel sich dennoch erfüllte? 
Die Angelegenheit hat eine subjektive und eine objektive Seite. Subjektiv muss man sich an Ödipus halten, bzw. an den Einzelnen, objektiv an den Zusammenhang der Geschichte, wie ihn die Schatten ihrer Protagonisten enthüllen. Die Handlung entwickelt sich durch das Zusammenspiel der Intentionen Einzelner im Dickicht von Missverständnissen und Korruption.

Die Antwort auf die subjektive Seite der Geschichte ist schon angedeutet worden. Die Erfüllung eines Schicksals braucht zwar keine Götter, aber doch einen gläubigen Menschen. Als einen solchen haben wir uns Ödipus vorzustellen. Zorn wird jedoch zur fatalen Kehrseite seines Glaubens, ein unbedingter Wille zur Rebellion, in dessen Hintergrund die alte Theodizeefrage steht: Wie konnten die Götter ihm ein solches Schicksal auferlegen, zulassen, dass er als „hilfloser Säugling“ dem Tode ausgesetzt wurde. In seinem Totenbericht erfahren wir, dass Ödipus zwar wusste, nicht von den Korinthischen Herrschern abzustammen; die wahren Eltern aber waren ihm unbekannt und sollten durch den Gang zum Orakel ermittelt werden. In seinem Hass auf sein Schicksal will Ödipus den Gott durch die falsche, längst beantwortete Frage, ob er der Sohn seiner Eltern sei, provozieren, ,aus seinem göttlichen Versteck hervor[locken]“ (289), wie es heißt. Das Orakel als Annahme der Herausforderung durch Apollon deutend, schließt Ödipus nicht nur, dass der erste Mensch, den er tötet, sein Vater sein muss, sondern ebenfalls, dass er, um zu seinem Wissen zu kommen, sein Schicksal auch erfüllen, den Mord und den Inzest wirklich begehen muss. Durch das folgende Orakel, das den Tod des Königsmörders fordert, glaubt er den Sinn der alten Verheißung endgültig zu verstehen: Die Götter wollten ihn vernichten. ${ }^{6}$ Und die einzige Möglichkeit, die Ödipus hat, um demgegenüber doch zu triumphieren, ist, wie Camus' Sisyphos sein Schicksal weiter auf sich zu nehmen: Er blendet sich und streift fortan als Bettler umher, nicht jedoch, wie er sagt, um ,die Macht der Götter zu verherrlichen, sondern um sie zu verhöhnen“ (292). In der vom Schicksal diktierten Selbstzerstörung und in der gleichzeitigen Revolte gegen sie, so die Logik des Ödipus, zeigen sich Sieg und Niederlage der Götter zugleich. Die grausige Würde, die Ödipus sich vorbehält, liegt darin, zerschmettert zu werden und als Zerschmetterter den Urhebern zum ewigen Vorwurf zu gereichen.

Man darf hier nicht vergessen: Diese verzweifelte Perspektive, in der man deutlich die Logik der sich selbst erfüllenden Prophezeiung erkennt, ist diejenige des Ödipus. Von der objektiven Seite her bleibt die Frage dennoch bestehen, wie das alles passieren konnte. Hat doch Ödipus, obwohl er dessen

6 Dürrenmatt stattet seinen Ödipus sowohl mit der leichten Entflammbarkeit als auch mit dem Bewusstsein seiner makelhaften Geburt aus, die er bei Sophokles besitzt: „Furchtbar ist die Schmach, die ich in den Windeln bekam" (Sophokles: König Ödipus, hrsg. von Jean Bollack. Bd. 1: Text, Übersetzung, Kommentar. Frankfurt am Main 1994, Vers 1035), so klagt er dort und glaubt später ebenfalls den Sinn seines frühen Überlebens zu erkennen: „Ich wäre sonst niemals vom Tode errettet worden, wäre es nicht in der Aussicht auf ein furchtbares Unglück geschehen."(Sophokles 1994, Vers 1456 f.) 
Identität nicht kannte, nicht nur irgendeinen Reisenden umgebracht, sondern seinen Vater, und die Frau, die er ehelichte, war seine Mutter. Wenn Dürrenmatt an dieser Stelle den Zufall bemüht, um die verhängnisvolle Begegnung objektiv zu motivieren, erweist sich dann nicht die Ersetzung von Unwissen durch Wissen, von Schicksal durch Zufall als allzu gewaltsam und die Erzählung als gescheitert? Verfolgen wir die Geschichte vorerst weiter.

Iokaste, so erfahren wir in ihrer Schattenrede, hatte an Ödipus' Füßen sofort bemerkt, mit wem sie im Bett lag, ${ }^{7}$ obwohl er ihr, sich verstellend, von der glücklichen Entscheidung erzählt hatte, nicht nach Korinth zurückzugehen und so dem verhängten Schicksal entkommen zu sein. Auch Iokaste jedoch war gläubig, und sie war überzeugt, Ödipus nicht aufklären zu dürfen, „um nicht gegen den Ratschluss der Götter zu handeln“ (293). Ebenso wenig aber erfährt Ödipus von Iokaste, dass in Wirklichkeit nicht der zeugungsunfähige Laios, sondern der Gardeoffizier Mnesippos sein Vater war - Mnesippos, den Ödipus, ohne viel dabei zu denken, gleich bei seiner Ankunft in Theben getötet hatte.

Diese erneute Korrektur verschiebt nicht nur die Konstellationen des Mythos, sie enthüllt auch, dass die zentralen Begriffe Wissen und Zufall nicht so unproblematisch sind, wie es schien. Dass Iokaste ebenfalls wusste, ahnte Ödipus nicht einmal, hielt er sich doch für den einzigen Wissenden. Ebenfalls als Irrtum erweist sich sein vermeintliches Wissen, in Laios den Vater ermordet zu haben, wenn er diesen auch unwissentlich in Mnesippos ermordet hat. Das Wissen des Ödipus relativiert sich. Der zunächst unglaubwürdige Zufall, der zum Mord an Laios führt, wird einerseits ebenfalls relativiert, da er gar nicht das vermeintliche Schicksal des Ödipus erfüllt, andererseits aber noch überboten durch den zweiten, im Sinne des Orakels ,richtigen“ Mord, den Ödipus ironischerweise unwissend begeht.

Erscheint dem Leser die Geschichte gerade durch die Überbietung plausibler, weil durch sie Wissen und Zufall paradox werden, so bringt die Sphinx eine weitere Drehung der Schraube. Ödipus, so berichtet sie, hatte nicht nur ihr Rätsel gelöst, sondern war auch ihr Geliebter geworden. Kein löwenleibiges Ungetüm ist sie, vielmehr eine wunderschöne Priesterin. Und als uneheliche Tochter des Laios und der Hippodameia gehört sie zum Geschlecht der Kadmiden. Aus Rache belagert sie die Stadt ihres Vaters. Denn dieser hatte einst, um die Weiterführung der Linie besorgt, seine Tochter durch den Wagenlenker Polyphontes vergewaltigen lassen, damit sie einen Enkel gebäre. Später wollte Laios sie aus Furcht vor dem Orakel zwingen, das Kind umzubringen. Nur zur Sicherheit ließ er ihr ebenfalls den Sohn der Iokaste und des Mnesippos aushändigen. Die Sphinx jedoch, unwillig zu diesem Opfer, tötet

7 Dürrenmatt ,korrigiert' hier einen der bekannten flaws des Oidipus Tyrannos, nämlich die Frage, warum Iokaste aufgrund von Oidipus’ Füßen nicht schon bei seiner Ankunft Verdacht geschöpft hat. 
das andere Kind und schafft ihren Sohn in das Gebirge: Ödipus. Der erste unter dem Gefolge des Laios aber, den dieser neue Ödipus zwischen Daulis und Delphi erschlug, war der Wagenlenker Polyphontes.

Die nunmehr dreifache Erfüllung des Orakels wird durchgehend von Korruption und dem Versuch, ihr entgegenzuarbeiten, flankiert. Angelpunkt dieser Aktivitäten ist Delphi. Mit dem frühesten der drei Orakel wollte Menoikeus seinen Sohn Kreon an die Regierung bringen. Die Formulierung dazu stammt jedoch von Tiresias, der den blinden Seher nur spielt, um die Politik trotz Machtgier und Korruption der Herrschenden ins Vernünttige zu lenken. Tiresias fürchtet Kreon als potentiellen Diktator. Das Orakel sollte Laios einen Hinweis geben, dass dieser, mittlerweile zeugungsunfähig, einen besseren Herrscher aus einem anderen Geschlecht berufen sollte - aber, so muss Tiresias erkennen, „Laios war nicht so klug, wie ich dachte“ (297). Die politische Einflussnahme endet fatal, sie erst ermöglicht den Aufstieg Kreons, indem sie die Voraussetzungen für die Handlung um Ödipus schafft, von dessen Existenz Tiresias nichts wusste. Das spätere Pestorakel gehorcht derselben Logik. „Jetzt gilt es, Ordnung zu schaffen“ (301) und Ödipus' Herrschaft gegen Kreon zu sichern - so denkt sich Tiresias, dem Iokaste inzwischen ihre Geschichte gebeichtet hat. Er geht jedoch davon aus, dass Kreon Laios aus dem Weg geräumt hat. Nach Tiresias' Intention hätte Kreon an Ödipus' Stelle vor dem Palast stehen und seine eigene Schuld ans Licht bringen sollen. Es kommt, wie wir wissen, anders.

Ironischerweise ist Tiresias erst als Sterbender ,sehend' geworden, so sehr, dass er auch nach dem Auftritt der Sphinx bezweifelt, die ganze Geschichte zu kennen, denn, so gibt er der sterbenden Pythia kurz vor ihrem Tod zu verstehen, ,vielleicht gibt es einen dritten Ödipus. Wir wissen nicht, ob der korinthische Hirte statt den Sohn der Sphinx [...] der Königin Merope seinen Sohn ausgeliefert hat, nachdem er [...] den echten Ödipus, der ja auch nicht der echte war, den wilden Tieren ausgesetzt hatte, oder ob Merope den dritten Ödipus nicht ins Meer geworfen hat, um ihren eigenen Sohn, den sie heimlich geboren $[\ldots]$ dem treuherzigen Polybos als vierten Ödipus zu präsentieren?“ (310 f.)

\section{Behauptung des Mythos durch seine Korrektur}

Das objektive Geschehen und das Wissen nicht nur der Beteiligten, sondern auch des Lesers sind vollständig auseinander gebrochen. Ödipus glaubt lediglich zu wissen, was er tut. Gegenüber den Zusammenhängen, wie sie Iokaste oder die Sphinx berichten, ist er jedoch so unwissend wie sein Sophokleischer Doppelgänger. Seine Überzeugung, an ihm sei das verheißene Schicksal erfüllt worden, und die unbekömmliche Energie, die ihn zu seiner 
Verstellung treibt, basieren auf einem Irrtum. Genauso Iokaste: ihr Glaube, mit dem Sohn und Vatermörder geschlafen zu haben - alles falsch. Tiresias schließlich führt durch seine tragischen Fehleinschätzungen jeweils das Gegenteil von dem herbei, was er intendiert hatte.

Die Korrekturen, denen Dürrenmatt die Geschichte von Ödipus unterzieht, liegen damit auf zwei Ebenen. Erstens verändert er grundsätzlich die Prämissen des Mythos wie auch des Sophokleischen Dramas, ersetzt Unwissen durch Wissen, Schicksal durch Zufall. Zweitens ergeben sich in der Erzählung selbst immer neue Berichtigungen der schon korrigierten Geschichte. Der Mythos wird zu einem fast undurchdringlichen Gefüge von vermeintlichem Wissen, politischer Wurstelei und Zufall. Durch diese Umschreibung aber - und hier zeigt sich eine dritte Ebene, auf der sich die Korrekturen bewegen - tritt Das Sterben der Pythia korrigierend in einen intertextuellen Bezug zu zwei anderen modernen Ödipus-Korrekturen. Die Möglichkeit eines wissenden Ödipus wurde durch Freuds Mythologisierung der Psychoanalyse angelegt und später von Brecht wieder aufgegriffen. Bei Freud weiß Ödipus zwar dezidiert nicht von seinem geheimen Triebwunsch, aber doch ist es dieser, der die innere, verborgene Logik der Figur wie des Mythos durchzieht. ${ }^{8}$ Die Polarität von Wissen oder Nichtwissen wird aufgebrochen, und die Tragik des Menschen entspringt aus der Grauzone zwischen beiden. Brecht formuliert in den Berichtigungen alter Mythen an einem solchen zugleich wissenden und nicht wissenden Ödipus ein modernes Konzept von Tragik: „Man hat immer gesagt: dies oder das brauche ich nicht zu befürchten, das kann nicht eintreten, es wäre zu unmenschlich. Dann tritt es ein, und all das, was menschlich ist, tritt in seinem ganzen Umfang auf, dem riesigen Umfang seines Schreckens".9 Ödipus wird zum Modell einer Verdrängungslogik, die die Tragik des modernen Menschen darin sieht, dass er das in ihm selbst Angelegte nicht erkennen will. Brechts Korrektur verleiht dem Mythos in seiner sophokleischen Bearbeitung eine geschichtliche und eine aufklärerische Dimension. Der Erzähler fingiert einerseits Naivität, wünscht sich, es hätte „dem Tragiker“ geziemt, „dem Zuschauer zuzublinzeln“10 und einzugestehen, dass Ödipus eigentlich doch etwas ahnt. Er gibt vor, das Drama des Sophokles unmittelbar, d. h. unhistorisch zu lesen, um diesem dann aus seinem Unverständnis heraus eine moderne, ,glaubwürdigere' Intention unterzuschieben. Indem er so zwar nicht die mögliche Lesart des König Ödipus ändert, aber doch die traditionsgeleitete Erwartung des Lesers konterkariert, wird die scheinbare Naivität des Gestus

8 Vgl. Sigmund Freud: Die Traumdeutung [1900]. Frankfurt am Main 1991, S. 268-272.

9 Bertolt Brecht: Berichtigungen alter Mythen. In: Ders.: Werke. Große kommentierte Berliner und Frankfurter Ausgabe, hrsg. von Werner Hecht u. a. Bd. 19. Berlin/Weimar/Frankfurt am Main 1997 (= Prosa 4), S. 340 f., hier S. 341. Die Berichtigungen entstanden zuerst 1933 unter dem Titel Zweifel am Mythos. Die zitierte Fassung datiert von 1954.

10 Brecht (1997), S. 341 [wie Anm. 9]. 
als Ironie sichtbar, hinter der sich wiederum eine aufklärerische Intention zu erkennen gibt.

Dürrenmatt verlängert diese geschichtliche Dimension der Brechtschen Berichtigung noch. Er korrigiert neben Sophokles auch die Ambivalenz des modernen Ödipus und unterzieht dadurch den modernen Wissensbegriff einer neuen Kritik. Mit Freud und Brecht teilt er die Annahme, dass es in unserer Zeit nicht mehr um Unwissen gehen kann, setzt dieses doch ein auf den Unwissenden bezogenes Schicksal voraus. Während Brecht aber die katastrophalen Folgen der Unaufrichtigkeit im Blick hat - und damit deren Vermeidbarkeit nahe legt -, formuliert Dürrenmatt, indem er Ödipus mit einem vollen Wissen ausstattet, global die Aporie jeden Weltzugriffs angesichts der unüberschaubar komplexen Zusammenhänge.

Die objektiven Fakten trennen sich damit in zweifacher Weise von der subjektiven Dimension des Wissens. Da erstens niemand um die ganze Geschichte weiß, ist adäquates Handeln von vornherein ausgeschlossen. Das gilt vor allem für Tiresias. Man könnte ihn als politischen Schriftsteller verstehen, der an die Kraft der Vernunft und an die Möglichkeit rationaler Veränderung durch seine Orakeldichtungen glaubt - und ihn geradezu als Kommentar zu Brecht lesen, den Dürrenmatt nicht umsonst als „Tiresias der materialistischen Dialektik" (320) bezeichnet. ${ }^{11}$ Der Glaube an die willentliche Veränderbarkeit der Welt endet in der tragischen Verkehrung der Intention in das Gegenteil. Zweitens aber legt die vom Objektiven her konstatierte Trennung von Wissen und Fakten dem Individuum nicht auf subjektiv notwendige Weise die Einsicht nahe, dass die Wirklichkeit unerkennbar sei. Denn der vorgängige Glaube oder das vorgängige Wissen haben eine pragmatische Dimension. Ödipus handelt nach seinem Glauben und bringt ihn damit zur Erfüllung, Iokaste ebenso. Niemand hat die Möglichkeit, seinen Irrtum einzusehen, und so wird das angenommene Wissen durch Handlung zur Realität, was die objektiven Verhältnisse jedoch nur noch mehr verwirrt.

Was für die handelnden Personen selber gilt, trifft auch auf den Versuch der Rekonstruktion zu. Nicht einmal von der ausgezeichneten Position der Pythia aus, die als Sterbende die Berichte der Toten entgegennehmen kann, erschließt sich die ,wirkliche" Version der Geschichte. Die wiederholten Mythenkorrekturen innerhalb der Handlung setzen eine mise en abyme des Mythos wie der Wahrheit ins Werk. Wenn Dürrenmatt das Geschehen umstrukturiert und mit modernen Prämissen ausstattet, so unterwandern die nachfolgenden Korrekturen diesen neu gewonnen Sinn wiederum. Wenn die immer neuen Perspektiven auf die Geschichte, je komplexer sie werden, desto mehr deren spekulativen Charakter enthüllen, dann bleibt am Ende ein menschliches Denken übrig, das vor der Unverständlichkeit der Welt nur noch

11 Jan Knopf bezeichnet sehr treffend Brecht als den von Dürrenmatt ,gesuchte[n] Antipode[n]“ (ders.: Friedrich Dürrenmatt. München ${ }^{4} 1988$, S. 158). 
kapitulieren kann. Die Analyse der sterbenden Pythia wird wieder und wieder über den Haufen geworfen, allein ihr Tod kann dem Prozess ein Ende setzen. Die gesuchte Wahrheit entpuppt sich als Abgrund, der immer noch tiefer ist als die in ihn herabgelassenen Leitern reichen.

Dem Sophokleischen Drama des unwissenden und der Brechtschen Tragödie des verdrängenden Ödipus setzt Dürrenmatt eine Farce des Wissens entgegen. Ihre Tragik liegt darin, dass sich auf jeder Stufe die schlimmstmögliche Wendung, Vatermord und Inzest, wiederholt, eine Tragik freilich, die sich, vom Ganzen aus gesehen, als bittere Ironie entpuppt. Der Zufall, der systematisch jede Erfüllung des Orakels sowohl bedingt und sie gleichzeitig als Illusion ironisiert, wird in der Welt des Erzählexperiments der Rolle des Schicksals ebenbürtig. Er bekommt eine eigene Art von Gesetzlichkeit: Unkontrollierbar, unvorhersehbar bricht er immer wieder in die Welt ein und schafft Fakten, die sich, von unterschiedlichen Charakteren unterschiedlich sinnhaft gedeutet, mit unerbittlicher Notwendigkeit fortzeugen. Sein einzig verlässlicher Zug scheint darin zu liegen, stets die schlimmstmögliche Wendung hervorzubringen. Dass das einzige wirklich nur dahinfabulierte Orakel, das der Pythia, sich unbeugsam immer wieder als Wahrheit erweist, ist die paradoxe Pointe der Geschichte. Der Mythos mag korrigiert werden, wie er will, sein fataler Ausgang behauptet sich doch. Aufklärung ist nicht in Sicht.

Wenn in Brechts Mythenkorrektur die aufklärerische Intention aus der Ironie hervorgeht, so verhält es sich bei Dürrenmatt umgekehrt. Schon die dreifache Korrektur: - die der Grundsituation, die Korrekturen dieser Korrektur, schließlich die intertextuelle Korrektur moderner Berichtigungen - setzt als paradoxe mise en abyme, als wiederholte Spiegelung, eine strukturelle Ironie ins Werk. Am Ende des Aufklärungsversuchs steht entsprechend ebenfalls eine ironische Einsicht: Die Welt ist ein Labyrinth ${ }^{12}$, dem der Mensch, der erforschend in ihm herumirrt, lediglich immer neue Windungen anfügt. Die Korrekturen des Mythos verschaffen zwar keinen Überblick über das Labyrinth; aber sie können zur Erkenntnis der Aporie verhelfen, zum Innehalten und zu dem Eingeständnis, dass die höchste Form des Wissens nur darin liegen kann: - zu erkennen, dass man eigentlich nicht weiß.

12 Das Labyrinth und der Mythos vom Minotauros sind in der gleichnamigen Ballade ( $\mathrm{F}$. Dürrenmatt: Minotaurus. Eine Ballade. Zürich 1985) und im Winterkrieg in Tibet (in F. Dürrenmatt: Stoffe I-III. Zürich 1981, S. 9-190) zentral. 
